I have found that these sub-titles, located in the candidate's head rather than on paper, are a valuable aid to clear thinking and make a serious omission less likely.

MORRIS Fraser

University College Hospital

London

(This correspondence is now closed-Ed.)

\section{Working party on psychiatric tutors}

\section{DeAR SIRS}

I write to inform readers that the Trainees' Committee of the College has recently set up a Working Party to examine problems in the present system of Psychiatric Tutors. Already the role of the Tutor is essential to the development of a good training scheme, and proposals have been put forward which would give the Tutor a greater role in the training of individuals (e.g. in sponsorship for the examination). However, there have been reports that, in addition to other problems, some tutors have had difficulties in finding sufficient time from clinical work to perform their tutorial duties adequately.

We would be grateful to receive comments from tutors or trainees about any problems which they have encountered with the scheme. These may be in the form of ideas for changes to the system generally, or in the form of specific experiences. If you wish to communicate with the working group, please write to the convener, Dr Chris Thompson, at the Maudsley Hospital, Denmark Hill, London SE5.

CHRIS THOMPSON

Maudsley Hospital

London SE5

\section{Physical Treatment Units}

DeAR Sirs

It was interesting to read Dr. White's letter (Bulletin, July 1983, 7, 128) describing the setting up of a Physical Treatment Unit and the part that this played in the rehabilitation of long-stay patients. Working as a registrar in a general psychiatry unit, I have had responsibility for a large longstay ward, and we have attempted to operate a similar system for the past six months.

In the hospital concerned, Middlewood, we already had a clinic that is visited by the physicians from a local general hospital. This facility is, however, primarily for the purpose of dealing with medical problems, and being an extension of out-patient facilities, it does not cater for the "crop of physical ailments' mentioned by $\mathrm{Dr}$ White.

We therefore set up a 'GP surgery' on the ward. This was manned by a local GP, who had been contracted to do some clinical assistant sessions. It had been noticed that the ward residents always referred to the nursing staff whenever they had a problem or request. This surgery was part of an attempt to encourage patients to seek out the appropriate agency to help them with a problem, in this case a doctor, rather than going to the nurses and having everything arranged for them. The provision of a local GP, working as a GP, offered a simulation of life outside the hospital environment.

In the six months that the scheme was in operation, it was not entirely successful. Residents did not, on the whole, attend the clinic spontaneously, but had to be taken along by the nursing staff. The one resident who did come along spontaneously appeared to see the exercise as an opportunity to harangue someone new, rather than to get physical problems solved.

Due to changes in medical and nursing staff rotations, it has become necessary to stop the scheme. In retrospect, I feel that a slightly different approach would have increased the likelihood of success. A single ward population is perhaps too small to give the system a chance to work; a system covering a group of wards or the entire hospital would be more appropriate. If the problems of available space and staff could be overcome, a site outside the ward environment should be sought for the clinic. I also feel that six months is not sufficient time for long-stay patients to get used to such a scheme.

In conclusion. I feel that this experiment has been a worthwhile exercise, and provides useful experience for further ventures of a similar kind.

Middlewood Hospital

P. GILL Sheffield

\section{Consent to treatment in the Mental Health Act}

\section{DeAR Sirs}

Bridgit C. Dimond's article (Bulletin, August 1983, 7, 145) was very interesting and thought-provoking, but I wonder whether anyone could enlighten me on a further point. Under the Mental Health Act (1959) it appeared that, strictly speaking, nobody was able to give consent to an operation for any non-lifethreatening condition occurring in a severely subnormal patient over the age of 16: by definition, the patient himself was incapable of giving valid (i.e. informed) consent, but being over the age of 16 , his parents/guardians were not entitled to do so either. Has anything been done to rectify this or is the situation as bad (or worse, which is the usual result of 'improving' legislation) as ever?

Mount Pleasant Hospital

P. J. H. GosLing

Ore, Hastings 